CARE-Management konkret bedeutet:

- systemspezifisches Management

- Öffentlichkeitsarbeit

- interdisziplinäre Arbeitskreise

- interdisziplinäre Fort- und Weiterbildung

- Care- und Pflegeberatung

- Koordinationsstelle für Kommunen und Träger

- Leitstelle für Profis

- Wegweiser

- Internet, Information

Das ganzheitliche Care Management umfasst verschiedene Bausteine (vgl. Abb.2).

Case Management konkret bedeutet: - einzelfallspezifisches Management

- Screening, Assessment

- personenzentrierte Fallbesprechung

- personenzentrierte Informationen und Dokumentation

- EDV-Konzept »Case Management «

- Angehörigenunterstützung

In ganzheitlichen Ansätzen der Gesundheitsförderung liegen erhebliche Potenziale für stationäre Einrichtungen und ambulante Dienste der Altenpflege. So bietet sich die Verknüpfung zu stärker »konsum- und genussorientierten « Dienstleistungen an, das können beispielsweise Präventions- und Sportangebote sein. Die Kopplung mit solchen Dienstleistungen wie Wellness-Angeboten verhilft der Seniorenwirtschaft zu einer Imageverbesserung, die auch unter wirtschaftlichen Gesichtspunkten dringend notwendig ist. Präventionsund Aufklärungsleistungen lassen sich gut mit anderen Dienstleistungen, wie Pflegeberatung und Wohnraumanpassung, koppeln. Gleiches gilt für die Bedürfnisse einer seniorenspezifischen Ernährung und Gesundheit sowie eine Angebotspalette von haushaltsnahen Dienstleistungen.

\title{
Integriertes Management in der Altenhilfe
}

Bei der Lektüre der Vorschläge von Andreas Tietze sind mir drei Gesichtspunkte aufgefallen, die ich kurz skizzieren möchte:

1 Von den 1. Schwierigkeiten der Normalisierungsarbeit: Ich erinnere mich an eine Begegnung mit Alexander Mitscherlich im Jahr 1969. Im Kontext einer Projektarbeit an der Architekturabteilung der Universität Stuttgart argumentierte er: »Es wäre zu empfehlen, sich nicht unbesehen an die Lösung des Problems >Altenheime $<\mathrm{zu}$ machen, wenn noch gar nicht feststeht, wo der Sinn solcher Heime liegt und wo der Unsinn anfängt. Das könnte $\mathrm{zu}$ leicht sich als einen Beitrag zur Katastrophe der falsch gehandhabten Wirklichkeit erweisen. « Mitscherlich wollte uns zukünftige Architekten motivieren, die Versorgung alter Menschen mehr unter dem Blickwinkel alltäglicher Haushaltung zu untersuchen und nicht der professionellen Verführung zur Hospitalisierung der Alten zu folgen. Diese Wirklichkeitskonstruktion stieß damals vor allem bei der kommunalen Altenhilfeplanung auf Widerstände. Dies wurde beispielsweise deutlich bei der Stuttgarter Diskussion »Altenhilfe 1971-1980«. Nun sind wir möglicherweise dort angekommen, wo wir damals Mitscherlich fol-gend hin wollten: ein gesetzlich abgesichertes Versorgungskonzept, das alltägliche und in der Nachbarschaft eingebundene Lebensführung als Ausgangspunkt der Planung achtet.

2. Von der Ver. führbarkeit der Planerinnen und Planer: Mitscherlich wolle uns dafür sensibilisieren, dass wir damit rechnen müssen, von der Gesellschaft falsche Aufträge zu erhalten, weil diese sich nicht genügend Rechenschaft gibt über das, was in ihr passiert also blind ist gegenüber dem, was die Alten wirklich wollen, aber dem folgt, was andere verdienen können, wenn man alte Menschen zur Ware erklärt und Profit zieht aus der Herrichtung entsprechender Versorgungsangebote. Er polemisierte gegen eine »Wohlfahrtsindustrie«, vor deren Verführungen wir uns als Planerinnen und Planer hüten sollten. In jedem »falschen « Auftrag beispielsweise in der Planung von Altenheimen - bestände die Möglichkeit des Widerspruchs, den wir nutzen könnten, um die technisch-industrielle Umwelt zu »humanisieren «. Keine einfache Aufgabe, der wir uns damals stellen wollten. Vielleicht ist es auch ein unmöglicher Auftrag gewesen, wie wir heute aus systemischer Perspektive formulieren könnten. Das System der Sozialen Arbeit und damit das System der Wohlfahrtsindustrie sei als »Funk- tionssystem der Gesellschaft « um seine eigenen Selbsterhaltung willen bewusstseinsfrei organisiert als ein System der Klientifizierung, weil es sich sonst nicht reproduzieren könne. Damals ging es eben um die Klientifizierung über das Angebot der Altenheime, was bis heute immer noch funktionieren kann - aber zunehmend schlechter funktioniert, weil die Kostenträger über Ambulantisierung und Sozialraumorientierung Spareffekte realisieren wollen, die als Nachfolgeeffekt »Humanisierung « bringen können. Es gibt Kollegen, die die Soziale Arbeit als eine Strategie in der Postmoderne beschreiben. Hier ginge es darum, Ambivalenzen auszuhalten, balancieren $\mathrm{zu}$ lernen zwischen »kritischem Bewusstsein « und »Arbeit in kritischen Zuständen«, also nicht dem Satz Adornos zu folgen, »dass es kein richtiges Leben geben könne im Falschen «, sondern die Gleichzeitigkeit von »richtig « und »falsch « zu ertragen und zu gestalten. Es könne keine Eindeutigkeit mehr geben, sondern nur ein System von Mehrdeutigkeiten. »Gestalten wird in diesem Theorie-Praxis-Konstrukt als Managementbalance zwischen Stabilität und Instabilität verstanden. Dort, wo Stabilität zugelassen wird, herrscht Eindeutigkeit, dort wo Instabilität unvermeidbar ist, herrscht Mehrdeutigkeit. Es ginge dann beispielsweise in der Altenhilfe um den 
Integrationsversuch verschiedener Teilprozesse, um eine Balance zwischen »individueller Unterstützung « (Case Management) und »Systementwicklung (Care Management). Diese Aufgabe kann als Versuch eines integrativen Managements in der Altenhilfe praktisch beschrieben werden.

3. Praxisperspektive für inte. griertes Altenhilfemanage-
ment: In dem Artikel von Andreas Tietze werden Zusammenhänge zwischen Care Management und Case Management dargestellt. Um dies zu verdeutlichen, empfiehlt sich eine Modellierung, die im Rahmen von Überlegungen zur lernenden Organisation erfolgreich erprobt wurden. Es kann davon ausgegangen werden, dass bei einem regionalen Altenhilfemanagement vier Teilprozesse integriert werden müssen:

- individuelle Unterstützung: das eigentliche Feld des Case Management (Teilprozess 1)

- regionales Controlling (Teilprozess 2), das eine summative Evaluation aller individueller Hilfepläne in einem festgelegten Zeitraum im Hinblick auf die Angebote der Versorgungsregion übernimmt

- regionale Planung (Teilprozess 3): Hier werden die Konsequenzen aus den Controlling-Ergebnissen umgesetzt in Verbesserungsvorschläge für die regionale Angebotspalette (Entscheidungsvorbereitung).

- regionale Politik (Teilprozess 4): Hier werden in den relevanten Entscheidungsgremien (z. B. im Kommunalparlament und den Trägerversammlungen) verbindliche Entscheidungen zur Fortentwicklung der regionalen Angebotspalette getroffen.

Die Aufgabe eines Pflegestützpunktes wird in der Integration der vier Teilprozesse gesehen - isofern handelt es sich um eine lernende Organisation: Sie sorgt für eine ständige Überprüfung und Anpassung der regionalen Angebote an sich verändernde Bedarfe. In jedem Teilprozess sollte es zum Informationsmanagement entsprechend besetzte Gremien geben, die - wenn es geht nach einem Prinzip der $»$ Allparteilichkeit « arbeiten. Beispielsweise

- im Teilprozess 1 Hilfeplankonferenzen

- im Teilprozess 2 Fachkonferenzen mit den Controlling-Mitarbeitern der beteiligten Stellen

- im Teilprozess 3 Planungswerkstätten mit den beteiligten Stellen und besetzt mit entsprechenden Planungsbeauftragten

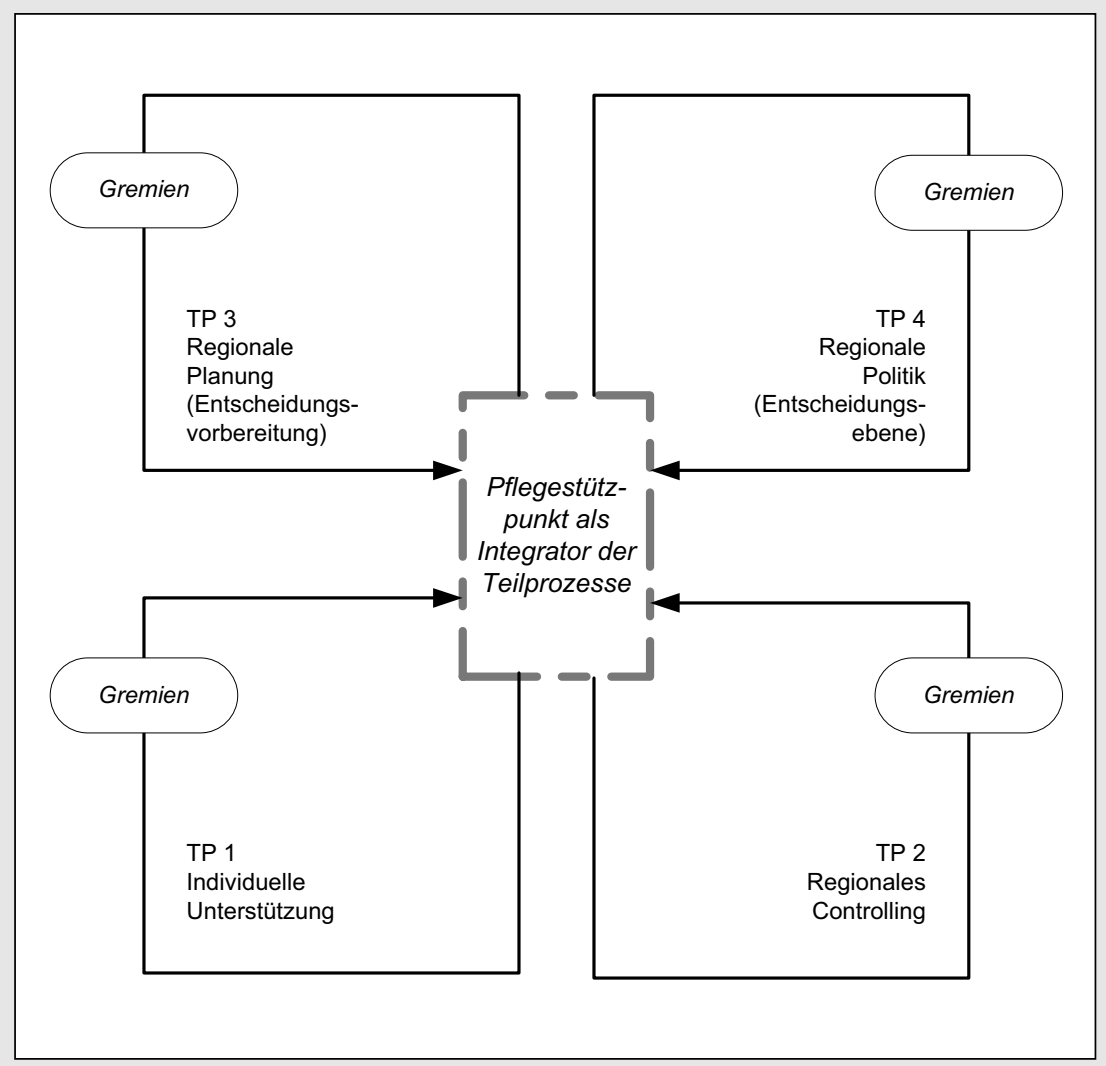

- im Teilprozess 4 Entscheidungsgremien aus dem öffentlichen und sozialwirtschaftlichen Bereich, die relevant sind für die regionale Politik

Idealtypisch ergibt sich daraus eine Modellierung des Gesamtsystems (vgl. Abbildung). Entlang dieser Modellierung müssen eine Reihe von Strukturentscheidungen getroffen werden, die das Funktionieren des Abstimmungs- und Entwicklungsprozesses ermöglichen; beispielsweise:

- Definition des Versorgungsraumes (»Region $«)$

- Festlegung verbindlicher Verfahrensregelungen

- Beschreibung der Managementtools im Pflegestützpunkt

- Ausarbeitung von Geschäftsordnungen der Gremien, sofern diese nicht schon aufgrund rechtlicher Vorgaben festliegen

- Fixierung von Gesichtspunkten der Personalauswahl und der Personalentwicklung für die verschiedenen Arbeitsplätze im Gesamtsystem

- Bestimmung relevanter und zu beachtender Schnittstellen zu den verschiedenen Sektoren des Unterstützungsund Versorgungssystems (z. B. Wohnungsmarkt)

- Sicherung eines effektiven Verbraucherschutzes in der Region

Der letztgenannte Punkt ist wichtig für die Frage der Ressortierung eines Pflegestützpunktes: Je besser der Verbraucherschutz in der Region funktioniert, desto flexibler ist man in der Frage der Ressortierung. Es gibt »ideologische « Festlegungen (»Ein Pflegestützpunkt gehört in die Hand der Kommune! $)$. Solche Festlegungen sind unnötig, wenn der Pflegestützpunkt sich als »Verbraucherlobby « verstehen kann. Eine solche Grundhaltung wäre wünschenswert, weil sie eine gewisse Garantie für eine nachfrageorientierte Steuerung der Altenhilfe gibt.

Prof. Dr. Andreas Strunk

Prof. Dr. Andreas Strunk ist Mitglied im Beirat der Zeitschrift SOZIALwirtschaft und des Informationsdienstes SOZIALwirtschaft aktuell ( $\mathrm{vgl}$. Autorenhinweise Seite 7).

E-Mail prof.strunk@t-online.de 